\title{
BMJ Open Medical versus surgical methods of early abortion: protocol for a systematic review and environmental scan of patient decision aids
}

\author{
Kyla Z Donnelly, Rachel Thompson
}

To cite: Donnelly KZ, Thompson R. Medical versus surgical methods of early abortion: protocol for a systematic review and environmental scan of patient decision aids. BMJ Open 2015;5:e007966. doi:10.1136/bmjopen-2015007966

- Prepublication history and additional material is available. To view please visit the journal (http://dx.doi.org/ 10.1136/bmjopen-2015007966).

Received 13 February 2015 Revised 12 May 2015 Accepted 13 May 2015

\section{CrossMark}

The Dartmouth Institute for Health Policy and Clinical Practice, Dartmouth College, Lebanon, New Hampshire, USA

\section{Correspondence to} Kyla Z Donnelly; kyla.z.donnelly@dartmouth. edu

\section{ABSTRACT}

Introduction: Currently, we lack understanding of the content, quality and impact of patient decision aids to support decision-making between medical and surgical methods of early abortion. We plan to undertake a systematic review of peer-reviewed literature to identify, appraise and describe the impact of early abortion method decision aids evaluated quantitatively (Part I), and an environmental scan to identify and appraise other early abortion method decision aids developed in the US (Part II).

Methods and analysis: For the systematic review, we will search PubMed, Cochrane Library, CINAHL, EMBASE and PsycINFO databases for articles describing experimental and observational studies evaluating the impact of an early abortion method decision aid on women's decision-making processes and outcomes. For the environmental scan, we will identify decision aids by supplementing the systematic review search with Internet-based searches and key informant consultation. The primary reviewer will assess all studies and decision aids for eligibility, and a second reviewer will also assess a subset of these. Both reviewers will independently assess risk of bias in the studies and abstract data using a piloted form. Finally, both reviewers will assess decision aid quality using the International Patient Decision Aid Standards criteria, ease of readability using Flesch/Flesch-Kincaid tests, and informational content using directed content analysis.

Ethics and dissemination: As this study does not involve human subjects, ethical approval will not be sought. We aim to disseminate the findings in a scientific journal, via academic and/or professional conferences and among the broader community to contribute knowledge about current early abortion method decision-making support.

Trial registration number: This protocol is registered in the International Prospective Register of Systematic Reviews (CRD42015016717).

\section{BACKGROUND}

In the USA, over 1 million women experience an induced abortion each year, ${ }^{1}$ and a

\section{Strengths and limitations of this study}

- Uniquely, this study combines a standard systematic review with an environmental scan much broader in scope.

- Our methodology will enable a comprehensive understanding of the early abortion method decision aids available to women and their content, quality and impact.

- Although informed by the broader goals of this work, one weakness of the study is that the environmental scan is limited to a search of decision aids developed in the USA.

majority $(64 \%)$ of these abortions occur during early pregnancy (ie, before 9 weeks' gestation). ${ }^{2}$ Over a decade ago, mifepristone was approved by the US Food and Drug Administration for medical management of early abortion, ${ }^{3}$ affording women a choice between medical and surgical procedures. ${ }^{i}$ These methods have comparable success rates, but encompass quite different processes. Accordingly, women's preferences and circumstances are paramount in the choice between early abortion methods. ${ }^{3}$ Some women favour medical abortion because they perceive it to be more natural, want to avoid surgery or value the privacy of the home environment. ${ }^{4-7}$ Other women prefer surgical abortion because of the comfort and finality of the procedure being completed in a single clinic visit, their preference for anaesthesia or their desire not to see the fetus. ${ }^{589}$

Research has documented the significant value women place on having a choice between medical and surgical abortion, ${ }^{7-11}$ and on receiving information to support their informed decision-making. ${ }^{12-14}$ Despite

${ }^{\mathrm{i}}$ Although both methods are approved federally, differences in state-level regulations has made access to early abortion services more variable. ${ }^{1}$ 
women's articulated desire for trusted resources, scant research has evaluated the content or quality of information available to facilitate women's early abortion method decision-making. Evidence from the UK indicates that some information materials designed for this purpose are inadequate, unbalanced and difficult to read. ${ }^{15}$ However, we are not aware of equivalent research in the USA or elsewhere. Such research is particularly important in the USA where abortion is simultaneously a highly prevalent and yet highly stigmatized ${ }^{16}$ procedure, and where there remains widespread misinformation, ${ }^{17}{ }^{18}$ poor access to services, ${ }^{1}$ and broad variability in abortion counselling practices.

Counselling about abortion methods has the potential to overcome shortcomings in the quality and comprehensiveness of information materials. However, although women in the USA tend to report satisfaction with abortion counselling generally, ${ }^{19}$ current information provision about available methods may limit informed, values-consistent decision-making. Indeed, there is evidence that some adolescent medicine providers have poor knowledge about the safety and effectiveness of medical abortion, ${ }^{20}$ and that some front-line health workers and social service providers feel they lack the necessary skills and information to adequately support women when considering early abortion. ${ }^{21}$ Studies have also found that healthcare providers have not disclosed information about available second trimester abortion ${ }^{22}$ or ectopic pregnancy treatment options, ${ }^{23}$ potentially delaying women's care or exposing them to unnecessary risk. It is unsurprising that in a recent qualitative study of 22 early medical abortion patients, women's beliefs about surgical abortion were often inaccurate. ${ }^{4}$ Similarly, a recent survey of 67 women who had recently experienced early surgical abortion found that $78 \%$ overestimated the health risks of the procedure. ${ }^{24}$

On the basis of the evidence described above, several authors have called for the development of evidencebased educational interventions to enable women to make quality and unbiased choices about early abortion methods. ${ }^{24}{ }^{25}$ One such intervention, the decision aid, is designed for this purpose. Specifically, decision aids are intended to help patients participate in making deliberative choices among healthcare options based on quality evidence and their preferences. ${ }^{26}$ Decision aids have been shown to improve patients' knowledge of options, accurate expectations of possible benefits and harms and participation in decision-making as well as the alignment between patients' choices and their values. ${ }^{26}$ Accordingly, implementation of a decision aid on early abortion methods has potential to address current deficiencies in information provision and counselling in the USA, particularly in areas with growing restrictions on abortion services. ${ }^{1}$

Three previous systematic reviews ${ }^{26-28}$ consistently identified only one study evaluating a decision aid on early abortion methods. This study, which was conducted in the UK in 2002, evaluated a one-page paper decision aid that described surgical and medical methods of early abortion. ${ }^{29}$ The study found that the 163 women randomised to use the decision aid had better knowledge and felt more informed than the 165 women randomised to receive usual care. ${ }^{30}$ While this study speaks to the potential of a decision aid in this clinical context, this particular tool has limited suitability for the contemporary US context due to sociopolitical and cultural differences in the provision of early abortion care ${ }^{31} 32$ and developments in evidence over the past decade. ${ }^{3}$ Whether other early abortion method decision aids-especially those relevant to the contemporary US context-have been developed and evaluated using non-randomised study designs, or have been developed but not evaluated quantitatively, remains unclear due to the scope of previous systematic reviews.

Ultimately, an inventory of available early abortion method decision aids, together with a summary of their quality and impact, would enhance our understanding of the decision support available to women choosing between medical and surgical early abortion. It would also elucidate necessary next steps for overcoming current limitations in information provision and counselling, and women's knowledge deficits, through implementation of a patient decision aid in the USA. The aim of this paper is to describe the protocol for a two-part study designed to identify existing decision aids and summarise evidence of their content, quality and impact.

\section{OBJECTIVES AND RESEARCH QUESTIONS}

This study will comprise a systematic review (SR) to identify, appraise and describe the impact of early abortion method decision aids that have been evaluated quantitatively and published in peer-reviewed outlets (part I) and an environmental scan (ES) of the grey literature to identify and appraise other early abortion method decision aids developed in the USA (part II). As this study does not involve human subjects, no ethical approval will be sought. This study will address several research questions (see table 1).

\section{METHODS: SYSTEMATIC REVIEW (PART I)}

This systematic review protocol was registered on 12 February 2015 with the International Prospective Register of Systematic Reviews (CRD42015016717) and adheres to PRISMA-P (Preferred Reporting Items for Systematic Reviews and Meta-Analyses protocols). ${ }^{33}$ Moreover, we intend to adhere to the PRISMA methodology when reporting on this systematic review. ${ }^{30}$

\section{Eligibility criteria}

Study design

Studies must be randomised controlled trials (ie, individual, crossover or cluster), non-randomised studies, cohort studies, case-control studies, before-and-after studies (ie, controlled or uncontrolled), interrupted-time-series studies or repeated measures studies. ${ }^{34}$ We will exclude 
Table 1 Study research questions

Research question

SR ES

What early abortion method decision aids have been evaluated quantitatively and published in peer-reviewed

outlets?

What other early abortion method decision aids have been developed for use in the USA?

$\checkmark$

What is the impact of early abortion method decision aids on women's decision-making processes and outcomes?

What is the quality of early abortion method decision aids as measured by the International Patient Decision Aid

Standards?

How does the content of early abortion method decision aids developed in the USA support quality

decision-making?

studies that do not have a comparison group (eg, case series, case reports).

\section{Participants}

Study participants must be women who are eligible for medical and surgical abortion (as defined by trialists) and who are facing a decision about method of early abortion. We will exclude studies involving women who are ineligible for medical and/or surgical abortion, who are making a hypothetical decision, or who are facing a decision about spontaneous abortion (ie, miscarriage).

\section{Intervention}

The intervention studied should have been a publicly available (ie, free) decision aid that compared medical and surgical abortion, and developed after 2000, when medical abortion became legal in the USA. ${ }^{35}$ Decision aids that include a third 'no treatment' option (ie, continuing the pregnancy or adoption) will be included if they also compare the two methods of early abortion. We will adopt the Cochrane Collaboration definition of a decision aid (see box 1). ${ }^{26}$

For the purpose of this review, implicit methods to clarify values' are defined as methods that prompt deliberation about options or attributes of options, ${ }^{36}$ such as a table enabling direct comparison of the features or outcomes associated with the options (eg, success rates, adverse effects) or a description of the options in "enough detail that clients can imagine what it is like to experience the physical, emotional, and social effects and/or guide clients to consider which benefits and harms are most important to them." ${ }^{26}$ The decision aid may be designed for use before, during, or after a healthcare appointment or independent of any healthcare appointment, and may be in any format (eg,

Box 1 Decision aid definition (Reproduced from Stacey et al, p. $8^{26}$ )

"(I)nterventions designed to help people make specific and deliberative choices among options (including the status quo), by making the decision explicit and by providing (at the minimum) (a) information on the options and outcomes relevant to a person's health status and (b) implicit methods to clarify values." pamphlets, booklets, videos, DVDs, interactive websites, static websites, electronic documents, smartphone mobile applications ('apps')). ${ }^{37}$ The decision aid may be published in any language and could have been created for women in any country.

We will exclude studies of interventions that do not meet the above definition of decision aid, including standard health education materials. We will also exclude interventions that are not publicly available and those not designed primarily for women facing the decision (eg, clinical guidelines).

\section{Comparison}

The comparison studied may comprise usual care, no intervention (or baseline) or a non-decision aid intervention. We will exclude studies that either do not include a comparison group or, in the case of beforeand-after studies, interrupted-time-series studies and repeated measures studies, that do not have a clearly defined intervention timeframe.

\section{Outcomes}

Studies must provide data on at least one outcome related to the decision-making process or decisionmaking outcome, whether reported by the woman, a provider and/or a third party (see table 2). Our primary outcome is decision quality, defined as the extent to which a decision is informed and based on personal values. ${ }^{38}$

\section{Date of study}

Based on the intervention inclusion criteria we will exclude studies published before 2000 .

\section{Data sources and search strategy}

We will search the following electronic bibliographic database: PubMed (see box 2), The Cochrane Library, CINAHL, EMBASE and PsycINFO using, where appropriate, medical subject heading $(\mathrm{MeSH})$ terms: "abortion, induced", "choice behavior", "patient education", "decision support techniques" and "decision making" and/or key words with Boolean operators (see online supplementary appendix 1). We will not apply any language limits. We will search for studies published since January 2000. We will also search the trial registry 
Table 2 Potential outcomes and their definitions

\begin{tabular}{|c|c|}
\hline Outcome & Definition \\
\hline Decision quality & The extent to which a decision is informed and based on the patient's personal values ${ }^{385556}$ \\
\hline Decision self-efficacy & A patient's degree of confidence or belief in one's ability to make decisions ${ }^{57}$ \\
\hline Decision aid utility & $\begin{array}{l}\text { The extent to which a decision aid is considered useful for preparing a patient to communicate } \\
\text { with his/her provider and to make a health decision }{ }^{58}\end{array}$ \\
\hline Decisional conflict & $\begin{array}{l}\text { A patient's degree of uncertainty about the right course of action to take (eg, due to to feeling } \\
\text { uninformed, unclear about personal values and/or unsupported in decision-making) }\end{array}$ \\
\hline $\begin{array}{l}\text { Decision-making role } \\
\text { concordance }\end{array}$ & The alignment between a patient's preferred and assumed decision-making role ${ }^{61} 62$ \\
\hline Shared decision-making & The extent to which a healthcare decision is made collaboratively by patient and provider ${ }^{63}$ \\
\hline
\end{tabular}

ClinicalTrials.gov and manually search the reference lists of all included articles for additional studies conducted or published since January 2000.

Additionally, any decision aid identified during the environmental scan (see below) that has been evaluated quantitatively and published in peer-reviewed outlets will be considered for inclusion in the systematic review.

\section{Data screening}

Search results will be downloaded and managed in Mendeley 1.13.1. After duplicate entries are removed, the primary reviewer (KD) will screen the titles and abstracts of all identified articles and classify each as potentially eligible or ineligible for inclusion. A second reviewer (RT) will independently screen random samples of $10 \%$ of the titles and abstracts in each classification. Inter-rater reliability will be measured using Cohen's $\kappa .{ }^{39}$ If significant discrepancies are observed in either classification (ie, $\kappa<0.7$ ), the second reviewer will screen all titles and abstracts, and discrepant classifications will be resolved by discussion.

\section{Full-text review}

The primary reviewer will perform full-text review of all potentially eligible articles and classify each as eligible or ineligible for inclusion. The reason for ineligibility will be recorded. If a classification cannot be made based on full-text review, attempts will be made to contact the study authors for clarifying information. The second reviewer will independently review a random sample of $10 \%$ of the full-text articles and inter-rater reliability will be assessed. If major discrepancies in classification are identified (ie, $\kappa<0.7$ ), the second reviewer will perform a

\section{Box 2 PubMed Search Strategy}

\section{Search Terms}

("abortion, induced" [MeSH] OR "pregnancy termination" [tiab] OR "termination of pregnancy" [tiab] OR "abortion" [tiab]) AND ("choice behavior" [MeSH] OR "decision support techniques" [MeSH] OR "patient education" [MeSH] OR "decision making" [MeSH] OR "decision support" [tiab] OR "decision aid" [tiab] OR "decision aids" [tiab] OR "informed choice" [tiab]) full-text review of all articles and discrepant classifications will be resolved by discussion. Finally, the primary reviewer will contact the author of each included study to inquire about any related studies (eg, study protocols, companion studies).

\section{Data abstraction}

Both reviewers will use a piloted form (see online supplementary appendix 2) to abstract data (ie, study design, participant characteristics, decision aid format and administration, findings, etc) from all studies deemed eligible for inclusion. We will also abstract data on other relevant outcomes measured by these studies. All abstracted data will be compared and disagreements resolved by discussion. If multiple publications describe a single study, authors will consider these as a single study.

\section{Study and evidence quality appraisal}

Both reviewers will independently assess the methodological quality of included studies using the Cochrane Effective Practice and Organisation of Care Group's (EPOC) criteria for randomised, non-randomised studies, before-and-after, interrupted-time-series studies or repeated measures studies ${ }^{40}$ and the NewcastleOttawa Scale for observational studies. ${ }^{41}$ Using EPOC criteria, we will apply a judgment of 'unclear risk', 'low risk' or 'high risk' to nine categories for each randomised, non-randomised and before-and-after study, and for seven categories for each interrupted-time-series and repeated measures study. ${ }^{40}$ Using the Newcastle-Ottawa Scale, we will award each study a maximum of nine points for items related to selection, comparability and outcome assessment bias. ${ }^{41}$

Both reviewers will also independently apply the Grading of Recommendations Assessment, Development and Evaluation (GRADE) criteria to rate the quality of evidence (high, moderate, low, very low) across studies for the primary outcome, decisional quality, using GRADEprofiler software (see online supplementary appendix 3). ${ }^{42}$ We will assess key factors that can downgrade the quality of evidence (eg, methodological quality, directness of evidence, heterogeneity, precision of effect estimates and risk of publication bias) and upgrade the quality of evidence (ie, magnitude of effect, 
dose-response gradient and ability of the study to limit bias and control for confounding). ${ }^{43}$ The quality of evidence can be downgraded or upgraded by one level or two levels to eventually describe a 'high', 'moderate', 'low' or 'very low' assessment of our confidence in the estimate of effect. The methodological and evidence quality assessments made by the reviewers will be compared and disagreements resolved by discussion.

\section{Decision aid quality appraisal}

Both reviewers will independently assess the quality of decision aids evaluated in included studies using the International Patient Decision Aid Standards (IPDAS) checklist. This tool has 59 items which evaluate the domains of content, development process and effectiveness for decision aids not involving tests. ${ }^{44}$ For each decision aid, we will assess each checklist item as 'yes' or 'no' or 'not applicable', with the final score ranging from 0 to 50 (Internet-based decision aids and those which integrate narratives have 6 and 3 additional items, respectively). Discrepant classifications will be resolved by discussion.

In addition, the primary reviewer will assess the readability and reading ease of text-based decision aids using the Flesch-Kincaid test. ${ }^{45}{ }^{46}$ The Flesch-Kincaid Grade Level measures textual difficulty based on words per sentence and syllables per word, and presents a score as a US grade level. The Flesch Reading Ease Scale uses average sentence length and the average number of syllables per word to calculate a score on a scale of 0 (very hard to read) to 100 (very easy to read).

\section{Analysis of decision aid impact}

We will quantitatively synthesise the findings from included studies if they are sufficiently homogeneous. We will calculate weighted treatment effects with $95 \%$ CIs. We will calculate risk ratios for dichotomous data and mean differences for continuous data. We will pool the results across studies with comparable outcome measures using a random-effects meta-analysis. When necessary data are available, we will compute standardised outcome scores to have a mean of 50 with SD of 10 for ease of descriptive comparison. If effect sizes cannot be calculated for the key outcomes due to inadequate information, we will contact the study authors for additional data. For each outcome, data will be synthesised and analysed using the statistical software, RevMan 5.1, as indicated by the Cochrane Handbook of Systematic Reviews of Interventions. ${ }^{47}$ Heterogeneity will be assessed and considered substantial if the $\mathrm{I}^{2}$ statistic is $>50 \%$ and the $\chi^{2}$ test $p$ value is $<0.10 .{ }^{48}$ If heterogeneity exists for a given outcome, we will seek to understand its source and conduct quantitative analysis on homogenous subgroups of studies. If relevant, the results of the methodological quality assessment will be used to perform sensitivity analyses to test the effect of omitting studies at high risk of bias. If meta-analysis is not possible, we will perform a narrative analysis and present summary information on all study characteristics and the primary outcome in text and tabular form.

We will explore the potential for publication bias visually using funnel plots if 10 or more studies are included. ${ }^{49}$ If study protocols have been published, we will also compare outcomes reported in the protocol and the published report to assess for selective outcome reporting.

\section{METHODS: ENVIRONMENTAL SCAN (PART II)}

Given the absence of quality standards for conducting environmental scan, our approach was informed by the methods of previous environmental scans about decision aids and/or shared decision-making. ${ }^{50} 51$

\section{Eligibility criteria \\ Intervention}

The intervention must be a decision aid, as described above. For the environmental scan only, the decision aid must be written in English and have been created by a source in the USA for women residing in the USA.

\section{Data sources and search strategy}

The Internet will comprise the primary data source for identifying decision aids for inclusion in the environmental scan. The Internet will be used both to identify relevant web-based decision aids and to identify references to non-web-based decision aids. We will execute four searches using the Google Advanced Search 'all these words' function and the following key word strings: (1) abortion decision aid, (2) abortion options, (3) pregnancy termination options and (4) medical or surgical abortion. We will select 'USA' in the 'region' category to refine the search. Cookies will be disabled before the search to avoid inadvertent bias. Efforts will be made to obtain any relevant documents that elaborate on the development or use of the decision aids. This may involve searching other sections of a multipage website and/or contacting the developers of the tools directly to request further information. To identify smartphone apps, we will search the two most frequently used Internet-based app stores, the English-language, USbased Apple app store and Google Play. ${ }^{52}$ We will use the key word abortion in the search field for both stores.

We will complement the Internet search by consulting organisations (eg, National Abortion Federation, Abortion Care Network, Reproductive Health Access Project, Planned Parenthood Federation of America, Provide and Society for Family Planning) and key informants (eg, women, researchers, clinicians, policymakers and/or advocates in the field). We will contact these organisations and individuals to solicit known examples of relevant decision aids (see online supplementary appendix 4) and will continue this consultation strategy until we determine that no new decision aids are likely to be identified. 
Additionally, any decision aid identified during the systematic review will be considered for inclusion in the environmental scan.

\section{Screening and full review}

Owing to the dynamic nature of the Internet, the screening and full review of Internet pages identified in the Google searches will be conducted concurrently. The primary reviewer will perform the first search string, archive the list of the first 100 results (ie, web address, page title, brief description and date searched), ${ }^{53}$ and classify each as potentially useful or not useful for identifying a decision aid. The reviewer will then open all pages considered potentially useful, archive each full page and if the page comprises or contains a decision aid, classify that tool as eligible or ineligible for inclusion. The reason for exclusion will be recorded. If the page contains only a reference to a decision aid, the primary reviewer will attempt to obtain a copy by contacting the source at a later time and, if successful, will then determine the eligibility of the decision aid for inclusion. Decision aids that cannot be obtained will be noted. This process will be replicated for each of the other three search strings.

For smartphone apps, the primary reviewer will archive and assess for eligibility the first 50 results on the general app description page of each Internet-based app store. The reviewer will then open, archive and assess the description of potentially eligible apps. The reviewer will download all apps considered potentially eligible to a smartphone and then classify each as eligible or ineligible for inclusion. At each stage, the reason for exclusion will be recorded.

The primary reviewer will use the same screening and review process to assess the eligibility of decision aids suggested by key informants.

Using the archives created by the primary reviewer, the second reviewer will independently open and assess random selections of $10 \%$ of the websites, apps and subsequently sourced decision aids classified by the primary reviewer as eligible and $10 \%$ of those classified as ineligible. The second reviewer will also archive the full page of each website opened for assessment (ie, included and excluded) in case the content has been modified since the primary reviewer undertook screening and full review. Inter-rater reliability will be measured using Cohen's $\kappa .{ }^{39}$ If significant discrepancies are observed in either classification (ie, $\kappa<0.7)$ that are not due to dynamic content, the second reviewer will complete screening and full review for all websites, apps and subsequently sourced decision aids, and the discrepant classifications will be resolved by discussion.

\section{Data abstraction}

Both reviewers will use a piloted form to abstract data from all decision aids (eg, characteristics, format and source) that meet inclusion criteria (see online supplementary appendix 5).

\section{Decision aid quality appraisal}

Both reviewers will independently evaluate the quality of the decision aids using the IPDAS checklist and the Flesch-Kincaid test, as described above.

\section{Decision aid content analysis}

We will analyse the content and presentation of information in included decision aids using methods from directed content analysis ${ }^{54}$ with an emphasis on distinguishing similarities and differences across the body of included tools. We will focus on identifying patterns in content and presentation relevant to promoting quality decision-making such as inclusion or omission of particular method features, word choice, framing of language about women's decision-making role and values clarification approaches. Data collection and analysis will follow an iterative process, whereby the coding structure and emerging themes will continuously be expanded and refined as more data is collected and analysed. The primary reviewer will lead the qualitative data collection and analysis process, and intermittently consult with the secondary reviewer with respect to the coding structure and emergent themes.

\section{CONCLUSION}

This protocol describes a study that will provide evidence about the content, quality and impact of early abortion method decision aids, and produce an inventory of appraised tools relevant to the US context. We intend to publish the results in academic and nonacademic outlets to contribute knowledge about current early abortion method decision-making support. In doing so, we hope to elucidate relevant next steps for attempting to overcome current limitations to counselling and women's knowledge deficits through implementation of a patient decision aid in the US.

Acknowledgements The authors are grateful to Glyn Elwyn for his guidance in the decision aid quality assessment strategy, to Greg McHugo for his input in the methods of the systematic review and to Heather Blunt for assisting in developing the search strategies.

Contributors KZD is the guarantor. KZD led the conception and design of the study and drafted the manuscript. RT contributed to the design of the study and provided revisions on the draft manuscript. Both authors approved the final manuscript.

Funding The preparation of this manuscript research received no specific grant from any funding agency in the public, commercial or not-for-profit sectors.

Competing interests None declared.

Provenance and peer review Not commissioned; externally peer reviewed.

Data sharing statement No additional data are available.

Open Access This is an Open Access article distributed in accordance with the Creative Commons Attribution Non Commercial (CC BY-NC 4.0) license, which permits others to distribute, remix, adapt, build upon this work noncommercially, and license their derivative works on different terms, provided the original work is properly cited and the use is non-commercial. See: http:// creativecommons.org/licenses/by-nc/4.0/ 


\section{REFERENCES}

1. Jones RK, Kooistra K. Abortion incidence and access to services in the United States, 2008. Perspect Sex Reprod Health 2011;43:41-50.

2. Pazol K, Creanga AA, Zane SB, et al. Abortion surveillance-United States, 2009. MMWR Surveill Summ 2012;61:1-44

3. American College of Obstetricians Gynecologists Committee on Practice Bulletins-Gynecology. ACOG Practice Bulletin. Clinical management guidelines for obstetrician-gynecologists. Medical management of abortion. Obstet Gynecol 2001;97 (suppl):1-13.

4. Cappiello J, Merrell J, Rentschler D. Women's experience of decision-making with medication abortion. MCN Am J Matern Child Nurs 2014;39:325-30.

5. Robson SC, Kelly T, Howel D, et al. Randomised preference trial of medical versus surgical termination of pregnancy less than 14 weeks' gestation (TOPS). Health Technol Assess 2009;13:1-124.

6. Swica Y, Chong E, Middleton T, et al. Acceptability of home use of mifepristone for medical abortion. Contraception 2013;88:122-7.

7. Fielding SL, Edmunds E, Schaff EA. Having an abortion using mifepristone and home misoprostol: a qualitative analysis of women's experiences. Perspect Sex Reprod Health 2002;34:34-40.

8. Slade $\mathrm{P}$, Heke S, Fletcher J, et al. A comparison of medical and surgical termination of pregnancy: choice, emotional impact and satisfaction with care. Br J Obstet Gynaecol 1998;105:1288-95.

9. Shochet T, Trussell J. Determinants of demand: method selection and provider preference among US women seeking abortion services. Contraception 2008;77:397-404.

10. Moreau C, Trussell J, Desfreres J, et al. Medical vs. surgical abortion: the importance of women's choice. Contraception 2011;84:224-9.

11. Henshaw RC, Naji SA, Russell IT, et al. A comparison of medical abortion (using mifepristone and gemeprost) with surgical vacuum aspiration: efficacy and early medical sequelae. Hum Reprod 1994:9:2167-72.

12. Zapka JG, Lemon S, Peterson LE, et al. The silent consumer: women's reports and ratings of abortion services. Med Care 2001;39:50-60.

13. The Picker Institute. From the patient's perspective: quality of abortion care. 1999 May. http://kaiserfamilyfoundation.files.wordpress.com/ 1999/04/ppqofabortioncare.pdf (accessed 1 Dec 2014)

14. Cockrill K, Weitz TA. Abortion patients' perceptions of abortion regulation. Womens Health Issues 2010;20:12-19.

15. Wong SS, Bekker HL, Thornton JG, et al. Choices about abortion method: assessing the quality of patient information leaflets in England and Wales. BJOG 2003;110:263-6.

16. O'Donnell J, Weitz TA, Freedman LR. Resistance and vulnerability to stigmatization in abortion work. Soc Sci Med 2011;73:1357-64.

17. Richardson $\mathrm{C}$, Nash E. Misinformed consent: the medical accuracy of state-developed abortion counseling materials. Guttmacher Policy Rev 2006:9:6-11.

18. Bryant AG, Narasimhan S, Bryant-Comstock $\mathrm{K}$, et al. Crisis pregnancy center websites: information, misinformation and disinformation. Contraception 2014;90:601-5.

19. Gould H, Foster DG, Perrucci AC, et al. Predictors of abortion counseling receipt and helpfulness in the United States. Womens Health Issues 2013;23:e249-55.

20. Coles MS, Makino KK, Phelps R. Knowledge of medication abortion among adolescent medicine providers. J Adolesc Health 2012;50:383-8

21. Zurek M, O'Donnell J, Hart R, et al. Referral-making in the current landscape of abortion access. Contraception 2015;91:1-5.

22. Kerns J, Vanjani R, Freedman L, et al. Women's decision making regarding choice of second trimester termination method for pregnancy complications. Int J Gynaecol Obstet 2012;116:244-8.

23. Foster AM, Dennis A, Smith F. Do religious restrictions influence ectopic pregnancy management? A national qualitative study. Womens Health Issues 2011;21:104-9.

24. Littman LL, Jacobs A, Negron R, et al. Beliefs about abortion risks in women returning to the clinic after their abortions: a pilot study. Contraception 2014;90:19-22.

25. Ely GE. The abortion counseling experience. Best Practices in Mental Health 2007;3:62-74

26. Stacey D, Legare F, Col NF, et al. Decision aids for people facing health treatment or screening decisions. Cochrane Database Syst Rev 2014;1:Cd001431.

27. Dugas M, Shorten A, Dube E, et al. Decision aid tools to support women's decision making in pregnancy and birth: a systematic review and meta-analysis. Soc Sci Med 2012;74:1968-78.

28. Vlemmix F, Warendorf JK, Rosman AN, et al. Decision aids to improve informed decision-making in pregnancy care: a systematic review. BJOG 2013;120:257-66.
29. Wong SS, Thornton JG, Gbolade B, et al. A randomised controlled trial of a decision-aid leaflet to facilitate women's choice between pregnancy termination methods. BJOG 2006;113:688-94.

30. Moher D, Liberati $A$, Tetzlaff $\mathrm{J}$, et al. Preferred reporting items for systematic reviews and meta-analyses: the PRISMA statement. Int $J$ Surg 2010;8:336-41.

31. Gissler M, Fronteira I, Jahn A, et al. Terminations of pregnancy in the European Union. BJOG 2012;119:324-32

32. Guttmacher Institute. Laws affecting reproductive health and rights 2011 state policy review. 2011. http://www.guttmacher.org/ statecenter/updates/2011/statetrends42011.html (accessed 10 Nov 2014).

33. Shamseer L, Moher D, Clarke M, et al. Preferred reporting items for systematic review and meta-analysis protocols (PRISMA-P) 2015: elaboration and explanation. BMJ 2015;349:g7647.

34. Effective Practice and Organisation of Care (EPOC). What study designs should be included in an EPOC review and what should they be called? EPOC Resources for review authors. Oslo: Norwegian Knowledge Centre for the Health Services, 2014. http:// epoc.cochrane.org/epoc-specific-resources-review-authors (accessed 20 Jan 2015).

35. Pazol K, Creanga A, Zane SB. Trends in use of medical abortion in the United States: reanalysis of surveillance data from the Centers for Disease Control and Prevention, 2001-2008. Contraception 2012;86:746-51.

36. Fagerlin $\mathrm{A}$, Pignone $\mathrm{M}$, Abhyankar $\mathrm{P}$, et al. Clarifying values: an updated review. BMC Med Inform Decis Mak 2013;13(Suppl 2):S8.

37. Elwyn G, Scholl I, Tietbohl C, et al. "Many miles to go...": a systematic review of the implementation of patient decision support interventions into routine clinical practice. BMC Med Inform Decis Mak 2013;13(Suppl 2):S14.

38. Sepucha KR, Fowler FJ Jr, Mulley AG Jr. Policy support for patient-centered care: the need for measurable improvements in decision quality. Health Aff (Millwood) 2004;(Suppl Variation): Var54-62.

39. Banerjee M, Capozzoli M, McSweeney L, et al. Beyond kappa: a review of interrater agreement meaures. Can J Stat 1999;27:3-23.

40. Effective Practice and Organisation of Care (EPOC). Suggested risk of bias criteria for EPOC reviews? EPOC Resources for review authors. Oslo: Norwegian Knowledge Centre for the Health Services, 2014. http://epoc.cochrane.org/epoc-specific-resourcesreview-authors (accessed 10 Feb 2015).

41. Well G, Shea B, O'Connell S, et al. The Newcastle-Ottawa Scale (NOS) for assessing the quality of nonrandomised studies in meta-analyses. Canada: Department of Epidemiology and Community Medicine, University of Ottawa, 2013. http://www ohri.ca/programs/clinical_epidemiology/oxford.asp (accessed 1 Dec 2014)

42. Guyatt G, Oxman AD, Akl EA, et al. GRADE guidelines: 1. Introduction-GRADE evidence profiles and summary of findings tables. J Clin Epidemiol 2011;64:383-94.

43. Schünemann HJ, Oxman AD, Vist GE, et al. 12.2.1 The GRADE approach. Cochrane handbook for systematic reviews of interventions Version 5.1.0 [updated March 2011]. The Cochrane Collaboration, 2011.

44. Elwyn G, O'Connor A, Stacey D, et al. Developing a quality criteria framework for patient decision aids: online international Delphi consensus process. BMJ 2006;333:417

45. Flesch R. A new readability yardstick. $J$ appl Psychol 1948;32:221-33.

46. Graber MA, Roller CM, Kaeble B. Readability levels of patient education material on the World Wide Web. J Fam Pract 1999;48:58-61.

47. Higgins JPT, Deeks JJ, eds. 7.8 Managing data. Cochrane handbook for systematic reviews of interventions Version 5.1.0 [updated March 2011]. The Cochrane Collaboration, 2011.

48. Deeks JJ, Higgins JP, Altman DG. 9.5.2 Identifying and measuring heterogeneity. Cochrane handbook for systematic reviews of interventions Version 5.1.0 [updated March 2011]. The Cochrane Collaboration, 2011

49. Richards BL, Whittle SL, Buchbinder R. Neuromodulators for pain management in rheumatoid arthritis. Cochrane Database Syst Rev 2011;1:CD008921.

50. Aslakson RA, Schuster AL, Miller J, et al. An environmental scan of advance care planning decision AIDS for patients undergoing major surgery: a study protocol. Patient 2014;7:207-17.

51. Legare F, Politi MC, Drolet R, et al. Training health professionals in shared decision-making: an international environmental scan. Patient Educ Couns 2012;88:159-69.

52. BinDhim NF, McGeechan K, Trevena L. Assessing the effect of an interactive decision-aid smartphone smoking cessation application 
(app) on quit rates: a double-blind automated randomised control trial protocol. BMJ Open 2014;4:e005371.

53. Tsulukidze M, Grande S, Thompson R, et al. Patients covertly recording clinical encounters: threat or opportunity? A qualitative analysis of online text. PLoS One 2015;10:e0125824.

54. Hsieh HF, Shannon SE. Three approaches to qualitative content analysis. Qual Health Res 2005;15:1277-88.

55. Michie S, Dormandy E, Marteau TM. The multi-dimensional measure of informed choice: a validation study. Patient Educ Couns 2002;48:87-91.

56. Holmes-Rovner M, Kroll J, Schmitt N, et al. Patient satisfaction with health care decisions: the satisfaction with decision scale. Med Decis Making 1996;16:58-64.

57. O'Connor A. User manual-Decision Self-Efficacy Scale [updated 2002]. Ottawa: Ottawa Hospital Research Institute, 1995. http:// decisionaid.ohri.ca/docs/develop/User Manuals/UM Decision SelfEfficacy.pdf (accessed 10 Jan 2015).
58. Bennett C, Graham ID, Kristjansson E, et al. Validation of a preparation for decision making scale. Patient Educ Couns 2010;78:130-3.

59. O'Connor AM. Validation of a decisional conflict scale. Med Decis Making 1995;15:25-30.

60. Légaré $\mathrm{F}$, Kearing $\mathrm{S}$, Clay $\mathrm{K}$, et al. Are you SURE?: assessing patient decisional conflict with a 4-item screening test. Can Fam Physician 2010;56:e308-14.

61. Degner LF, Sloan JA. Decision making during serious illness: what role do patients really want to play? J Clin Epidemiol 1992;45:941-50.

62. Degner LF, Kristjanson LJ, Bowman D, et al. Information needs and decisional preferences in women with breast cancer. JAMA 1997;277:1485-92.

63. Elwyn G, Laitner S, Coulter A, et al. Implementing shared decision making in the NHS. BMJ 2010;341:c5146. 